\title{
Comportamento ingestivo e taxa de passagem de partículas em novilhas leiteiras de diferentes grupos genéticos submetidas a dietas com diferentes níveis de fibra ${ }^{1}$
}

\author{
José Carlos Pereira², Daniel de Noronha Figueiredo Vieira da Cunha ${ }^{3}$, Paulo Roberto \\ Cecon ${ }^{4}$, Elisa de Souza Faria ${ }^{5}$
}

\author{
${ }^{1}$ Parte da Dissertação de Mestrado apresentada pela quarta autora à Universidade Federal de Viçosa. \\ 2 Departamento de Zootecnia - UFV. Pesquisador do CNPq. \\ ${ }^{3}$ Doutorando em Zootecnia - UFV. \\ ${ }^{4}$ Departamento de Informática - UFV. \\ ${ }^{5}$ Mestre em Zootecnia - UFV.
}

RESUMO - O trabalho foi conduzido para avaliar a taxa de passagem de partículas do volumoso e o comportamento ingestivo de novilhas leiteiras de três grupos genéticos Holandes (H) x Zebu (Z): 7/8HZ, 15/16 HZ e Holandês Puro por Cruza (HPC), mantidas em confinamento e alimentadas com dietas, em mistura completa, com dois níveis (30 e 60\%) de fibra em detergente neutro (FDN), utilizando-se como volumoso o capim-elefante (Pennisetum purpureum, Schum., cv. Mineirão) picado. O experimento foi conduzido em esquema fatorial $3 \times 2$ (grupo genético $\times$ nível de fibra), em delineamento em blocos casualizados, com quatro repetições. O ensaio para determinação da taxa de passagem foi conduzido no final do período experimental, em delineamento inteiramente casualizado com três repetições. O tempo de retenção no rúmen-retículo (TRRR) foi 13,8\% menor e o tempo de retenção no ceco-cólon (TRCC), 13,9\% maior para a dieta com 30\% de FDN em relação à dieta com $60 \%$ de FDN. Para o nível de $60 \%$ de FDN na dieta, houve maior tempo gasto com alimentação, ruminação e mastigação total, enquanto a taxa de ingestão e a taxa de ruminação da MS foram menores. Quanto aos grupos genéticos, o TRRR foi $14,75 \%$ maior para as novilhas $7 / 8 \mathrm{HZ}$ em relação às novilhas HPC e o TRCC foi 28,2\% menor para as novilhas 7/8HZ. As novilhas HPC gastaram mais tempo em atividades de alimentação e mastigação, porém, com menores taxas de ingestão que novilhas $7 / 8 \mathrm{HZ}$.

Palavras-chave: capim-elefante, cinética ruminal, mastigação merícica, tempo de mastigação

\section{Ingestive behavior and particles passage rate in dairy heifers from different genetic groups fed diets with different levels of fiber}

\begin{abstract}
This work was carried out to evaluate the forage particle passage rates and ingestive behavior of dairy heifers from three genetic groups Holstein $(\mathrm{H})$ and Zebu $(\mathrm{Z})$ cattle (7/8HZ, 15/16HZ, Graded Holstein - GH), kept in feedlot regime and submitted to diets, in a total mixed ration, with two fiber levels (30 and 60\%) of neutral detergent fiber (NDF), using chopped Elephant grass (Pennisetum purpureum, Schum., cv. Mineirão) as forage. The experiment was conducted in a $3 \times 2$ factorial arrangement (genetic group $\times$ fiber level), in a completely randomized design, with four replicates. The digestive kinetic trial was determined in the end of the experimental period in a completely randomized design with three replicates. Rumen-reticulum retention time (RRRT) was 13.8\% lower and cecum-colon retention time (CCRT) was $13.9 \%$ higher for the diet with $30 \%$ NDF in relation to the diet with $60 \%$ NDF. There was a higher spending time with feeding, rumination and total chew, while the ingestive rate and rumination rate of DM were lower for the $60 \%$ NDF level in the diet. As for genetic groups, RRRT was $14.75 \%$ higher for the $7 / 8 \mathrm{HZ}$ heifers than for $\mathrm{GH}$, and CCRT was $28.2 \%$ lower for $7 / 8 \mathrm{HZ}$ heifers. Graded Holstein heifers spent more time in activities of feeding and chewing, however, with lower intake rate than $7 / 8 \mathrm{HZ}$ heifers.
\end{abstract}

Key Words: chewing time, elephantgrass, particles rate, ruminal kinetics, ruminating chew

\section{Introdução}

A fibra desempenha importante função no controle do consumo voluntário e, conseqüentemente, na ingestão de nutrientes (Allen, 2000), além de garantir a função ruminal e o crescimento adequado da microbiota nesse compartimento.

De acordo com Mertens (1997), entre os componentes da fração fibrosa, o nível de FDN pode ser muito útil na determinação dos limites mínimos de concentrado e volu- 
moso. Entretanto, segundo Van Soest et al. (1994), a utilização desta fração no balanceamento de rações é um procedimento altamente complexo, por não se tratar de uma entidade nutricionalmente uniforme.

As informações na literatura (Tomlinson et al., 1991; Serjsen \& Foldager, 1992; NRC, 2001) quanto aos níveis adequados de fibra nas rações para animais de rebanhos leiteiros são bastante variáveis.

Segundo o NRC (2001), para vacas alimentadas com alfafa ou silagem de milho como volumosos predominantes e fubá de milho como principal fonte de amido, o nível mínimo de FDN da ração deve ser de $25 \%$, ressalvando que, para que se garanta o metabolismo adequado no rúmen, $76 \%$ desta FDN deve provir de forragens.

Tomlinson et al. (1991) concluíram que níveis de fibra abaixo de 30\% na ração de novilhas leiteiras atuam na limitação do consumo por meio de mecanismos metabólicos. A limitação física afetaria a capacidade de consumo em níveis de FDN maiores que 40\%, mesmo com a utilização de diferentes volumosos. Entretanto, Allen (2000) sumarizando diversos estudos, observou declínio na ingestão de MS quando a concentração de FDN excedeu 25 \% da MS.

Segundo Shafer et al. (1981), a variação com relação às recomendações de níveis adequados de fibra para novilhas de rebanhos leiteiros pode ser atribuída, em parte, a diferenças em relação à capacidade ingestiva de animais de grupos genéticos heterogêneos. Entretanto, parte desta variação pode estar relacionada a aspectos qualitativos da fibra, uma vez que, para diferentes rações contendo concentrações semelhantes de FDN, há grande variação na ingestão de MS, pois outros fatores como digestibilidade, tamanho de partícula, densidade e taxa de passagem afetam o consumo.

A taxa de passagem da digesta pelo trato gastrintestinal tem significativo efeito sobre a degradação ruminal da fibra, especialmente em animais de alto desempenho, pois, quanto maior a taxa de passagem, menor a degradabilidade da FDN (Bezerra et al., 2004).

O estudo do comportamento ingestivo pode ser utilizado como ferramenta para explicar parte das variações na ingestão de alimentos. Segundo Van Soest (1994), uma série de fatores influencia o tempo de alimentação, de ruminação e de mastigação. Os tempos despendidos com atividades de alimentação (Albright, 1993; Sarmento, 2003), de ruminação (Beauchemin \& Buchanan-Smith, 1990; Polli et al., 1996) e de mastigação (Beauchemin, 1991) variam de acordo com a concentração de FDN da dieta. O tempo de mastigação/kg de MS da ração (Sarmento, 2003) ou o tempo de mastigação/ kg de FDN consumido (Allen, 1995) são medidas biológicas que podem ser utilizadas para avaliação de características físicas da forragem. Entretanto, existem poucas informações relacionando estas medidas a características físicas de forragens de clima tropical.

Objetivou-se neste trabalho avaliar a cinética de passagem de partículas e o comportamento ingestivo de novilhas de rebanhos leiteiros de três grupos genéticos alimentadas com dietas com dois níveis de fibra.

\section{Material e Métodos}

Foram utilizadas 24 novilhas de três grupos genéticos, provenientes de cruzamentos entre animais da raça holandesa Preto e Branco (H) e animais azebuados, com predominância da raça Gir (Z): 7/8HZ, 15/16HZ e Holandês Puro por cruza (HPC), com peso vivo médio de 375 kg e mantidas em regime de confinamento.

As novilhas foram selecionadas com base no peso vivo médio, escore de condição corporal entre 3,0 e 3,5 em escala de 1 (muito magro) a 5 (obeso), e distribuídas aleatoriamente em baias individuais de piso concretado, com $12 \mathrm{~m}^{2}$ por animal, e parcialmente sombreadas ( $6 \mathrm{~m}^{2}$ de sombra às $12 \mathrm{~h}$ ). As rações fornecidas foram preparadas na forma de mistura completa e continham capim-elefante (Pennisetum purpureum Schum. cv. Mineirão) como volumoso. A mistura do concentrado foi constituída de farelo de soja, fubá de milho, farelo de trigo, farelo de algodão e fosfato bicálcico e foi ajustada a cada período experimental de acordo com a análise químico-bromatológica prévia do volumoso. Essas rações foram balanceadas para conter aproximadamente 30 e $60 \%$ de FDN, com base na MS, e nutrientes para atender às exigências de PB, NDT e minerais, conforme NRC (2001), para ganho médio de $700 \mathrm{~g} / \mathrm{animal} / \mathrm{dia}$.

As proporções percentuais do capim-elefante e do concentrado (Tabela 1), com base na MS, na dieta FDN30 foram de 29 e $71 \%$ e na dieta FDN60 foram iguais (50:50\%).

As rações foram fornecidas duas vezes ao dia, às $10 \mathrm{e}$ 16 h, $60 \%$ na parte da manhã e $40 \%$ na parte da tarde. Para início do fornecimento, tomou-se como base o consumo voluntário inicial de MS, estimado de acordo com o NRC (2001), de 2,2\% do peso vivo médio dos animais e, durante o período de adaptação, ajustado para $10 \%$ de sobras para garantir ingestão à vontade das dietas pelos animais. $\mathrm{O}$ acesso irrestrito à água e à mistura mineral foi garantido diariamente para todos os animais.

Utilizou-se um período inicial de 14 dias para ajuste do consumo e adaptação dos animais e, em seguida, decorreram-se três períodos experimentais, cada um de 14 dias, para as coletas dos dados.

O consumo voluntário foi determinado pela diferença entre as pesagens do oferecido e das sobras. Diariamente, 
Tabela 1 - Composição químico-bromatológica média (\%MS) dos ingredientes e das dietas experimentais Table 1 - Average chemical-bromatological composition (\%DM) of the ingredients and experimental diets

\begin{tabular}{|c|c|c|c|c|c|c|c|}
\hline Item & $\begin{array}{l}\text { MS (\%) } \\
\text { DM (\%) }\end{array}$ & $\begin{array}{r}\text { FDN } \\
N D F\end{array}$ & $\begin{array}{r}\text { FDA } \\
A D F\end{array}$ & $\begin{array}{l}\text { LIG } \\
\text { LIG }\end{array}$ & $\begin{array}{c}\text { CEL } \\
\text { CEL }\end{array}$ & $\begin{array}{l}\mathrm{PB} \\
C P\end{array}$ & $\begin{array}{c}\mathrm{NDT}^{1} \\
T D N\end{array}$ \\
\hline \multicolumn{8}{|l|}{$\begin{array}{l}\text { Ingrediente } \\
\text { Ingredient }\end{array}$} \\
\hline Capim-elefante (Elephantgrass) & 22,86 & 78,15 & 45,91 & 5,05 & 38,25 & 7,95 & 53 \\
\hline Farelo de soja (Soybean meal) & 86,30 & 12,82 & 7,72 & 0,27 & 7,25 & 56,53 & 87 \\
\hline Farelo de trigo (Wheat meal) & 84,75 & 39,05 & 12,24 & 2,86 & 9,15 & 16,90 & 70 \\
\hline Farelo de algodão (Cottonseed meal) & 87,83 & 46,36 & 30,51 & 14,00 & 12,00 & 29,69 & 96 \\
\hline Fubá de milho (Ground corn) & 86,93 & 11,29 & 2,64 & 0,07 & 2,75 & 9,98 & 85 \\
\hline \multicolumn{8}{|l|}{ Dietas (Diets) } \\
\hline FDN30 (NDF30) & 44,59 & 32,93 & 16,73 & 1,85 & 14,17 & 15,45 & 72 \\
\hline FDN60 (NDF60) & 34,91 & 60,59 & 29,90 & 4,14 & 24,98 & 13,11 & 60 \\
\hline
\end{tabular}

${ }^{1}$ Valores obtidos em tabelas de composição de alimentos (Values obtained from feed composition tables).

foram coletadas amostras do volumoso, do concentrado, da ração em mistura completa e das sobras. Nas amostras compostas, para cada animal e período, foram determinados os teores de MS e PB, segundo Silva (2002), além de FDN, FDA, lignina, celulose e hemicelulose, segundo o método de análise seqüencial proposto por Van Soest et al. (1991).

A taxa de passagem de partículas pelo rúmen foi determinada utilizando-se como marcador o cromo-FDN ou cromo-mordente fixado à parede celular do volumoso, de acordo com Úden et al. (1980). Após a ingestão de 100 g da fibra marcada pelas novilhas, foram coletados $200 \mathrm{~g}$ de fezes, aproximadamente, nos intervalos de 0, 12, 24, 30, 36, 48, 72, 96 e 120 horas, conforme procedimentos descritos por Bürger et al. (2000a). O conteúdo de cromo nestas amostras foi determinado por espectrofotometria de absorção atômica com chama de ar-acetileno, conforme método descrito por Willians et al. (1962).

Para determinação da taxa de passagem da fase sólida e do tempo de retenção de sólidos no rúmen e nos intestinos, as curvas de excreção fecal do marcador foram ajustadas ao modelo exponencial bicompartimental proposto por Grovum \& Willians (1973):

$\mathrm{Y}_{\mathrm{Cr}}=\mathrm{Ae}-\mathrm{k1}(\mathrm{t}-\mathrm{TT})-\left(\mathrm{Ae}^{-\mathrm{k} 2(\mathrm{t}-\mathrm{TT}),}\right.$ em que $\mathrm{Y}_{\mathrm{Cr}}=0$, para $\mathrm{t}<\mathrm{TT}$

em que $\mathrm{Y}_{\mathrm{Cr}}=$ concentração do indicador nas fezes; $\mathrm{A}=$ parâmetro biologicamente indefinido; $\mathrm{k}_{1}$ = taxa de passagem no rúmen; $\mathrm{t}$ = tempo de amostragem do indicador; $\mathrm{TT}=$ tempo de trânsito ou estimativa do tempo em que o indicador apareceu pela primeira vez nas fezes; $\mathrm{k}_{2}=$ taxa de passagem de partículas no ceco-cólon proximal.

Os parâmetros expressando a dinâmica da fase sólida foram determinados de acordo com Colucci et al. (1990):

$$
\operatorname{TRRR}=1 / \mathrm{k}_{1} \text { e TRCC }=1 / \mathrm{k}_{2}
$$

em que TRRR (hora) = tempo de retenção no rúmen-retículo;
TRCC (h) = tempo de retenção no ceco-cólon proximal; $\mathrm{k}_{1}$ $(\% / h)=$ taxa de passagem de partículas no rúmen-retículo; $\mathrm{k}_{2}$ $(\% / h)=$ taxa de passagem de partículas no ceco-cólon proximal .

$$
\mathrm{TMR}=\mathrm{TRRR}+\mathrm{TRCC}
$$

em que TMR (h) é o tempo médio de retenção nos dois compartimentos.

$$
\mathrm{TRT}=\mathrm{TMR}+\mathrm{TT}
$$

em que TRT (h) representa o tempo de retenção total e TT (h), o tempo de trânsito.

Os tempos despendidos em alimentação, ruminação, ócio e outras atividades foram registrados uma vez por semana em cada período experimental, para todos os tratamentos, por meio de observação visual dos animais durante 24 horas, a cada 5 minutos, segundo Johnson \& Combs (1991).

O número de mastigações merícicas e o tempo de mastigação por bolo ruminal foram medidos por meio de observações distribuídas em três turnos ao dia, nos horários das 8 às 10h, 14 às $16 \mathrm{~h}$ e 18 às 20h, conforme metodologia descrita por Bürger et al. (2000b), para o cálculo do comportamento mastigatório médio diário.

Os componentes do comportamento ingestivo foram calculados segundo Polli et al. (1996):

$$
\mathrm{EAL}=\mathrm{CMS} / \mathrm{TAL}
$$

em que EAL (kgMS/h) = taxa de ingestão; CMS (kgMS/ dia) = consumo de MS; TAL $(\mathrm{h} /$ dia $)=$ tempo de alimentação.

$$
\begin{aligned}
\mathrm{ERU}_{\mathrm{MS}} & =\mathrm{CMS} / \mathrm{TRU} \\
\mathrm{ERU}_{\mathrm{FDN}} & =\mathrm{CFDN} / \mathrm{TRU}
\end{aligned}
$$

em que $E R U_{M S}(\mathrm{kgMS} / \mathrm{h})=$ taxa de ruminação em relação ao CMS; $E_{\text {FDN }}(\mathrm{kgFDN} / \mathrm{h})$ = taxa de ruminação em relação ao cFDN; TRU (h/dia) = tempo de ruminação.

$\mathrm{TMT}=\mathrm{TAL}+\mathrm{TRU}$

em que TMT $(\mathrm{h} /$ dia $)=$ tempo de mastigação total.

$$
\mathrm{BOL}=\mathrm{TRU} / \mathrm{MM}_{\mathrm{tb}}
$$

em que BOL (no/dia) = número de bolos ruminais por dia; 
$\mathrm{MM}_{\mathrm{tb}}$ (s/bolo) = tempo de mastigação merícica por bolo ruminal, em segundos; TRU (s/dia) = tempo de ruminação, em segundos por dia.

O número de mastigações merícicas por dia foi estimado conforme Bürger et al. (2000b):

$$
\mathrm{MM}_{\mathrm{nd}}=\text { BOL x MM } \mathrm{nb}
$$

em que $\mathrm{MM}_{\mathrm{nb}}\left(\mathrm{n}^{\mathrm{o}} . / \mathrm{bolo}\right)=$ número de mastigações por bolo ruminal; $\mathrm{MM}_{\mathrm{nd}}\left(\mathrm{n}^{\circ} . / \mathrm{dia}\right)=$ número de mastigações merícicas por dia.

Os fatores do comportamento ingestivo e mastigatório foram avaliados ainda em relação ao consumo de matéria seca (h/kgMS) e ao consumo de FDN (h/kgFDN), conforme Beauchemin \& Buchanam-Smith (1990). A taxa de ruminação/kg de FDN consumido em relação tempo médio de retenção ruminal do FDN foi obtida com base nos dados estimados para a passagem da fibra marcada. A taxa de ruminação em relação ao tempo de mastigação total foi obtida de acordo com a equação:

$$
\mathrm{TR}=\mathrm{TRU} / \mathrm{TMT} \times 100
$$

em que TR $(\% / h)$ = taxa de ruminação em relação ao tempo de mastigação total.

O experimento foi conduzido em delineamento em blocos casualizados, em um esquema fatorial $3 \times 2$, com quatro repetições. Os dados de consumo e comportamento ingestivo foram analisados por meio de análise de variância e as médias comparadas pelo teste Tukey, adotando-se os níveis de 1 e 5\% de probabilidade. Para a determinação da cinética de trânsito, os animais foram distribuídos em delineamento inteiramente casualizado, em arranjo fatorial $3 \times 2$ com quatro repetições por tratamento. As estimativas das variáveis foram avaliadas fixando-se os níveis de fibra e variando-se os grupos genéticos. Efetuaram-se também análises para cada nível de fibra, independentemente do grupo genético. As análises estatísticas foram realizadas com a utilização do software SAEG (Euclydes, 1983).

\section{Resultados e Discussão}

As curvas de excreção fecal (Tabela 2) apresentaram maiores diferenças entre os grupos genéticos quando as novilhas foram alimentados com a dieta com baixo nível de fibra e tenderam a se aproximar quando os animais foram alimentados com a dieta com alta fibra. A estimativa da cinética de partículas não tem levado a resultados consistentes, em razão das variações encontradas conforme o local de amostragem, as respostas individuais de cada animal, além das alterações do abomaso e do ceco nos resultados obtidos nas fezes (Cruickshank et al., 1989). Não houve diferença $(\mathrm{P}>0,05)$ entre as dietas e entre os grupos genéticos para taxa de passagem e tempo de retenção. As pequenas diferenças nos valores das taxas de passagem estimadas neste trabalho, para as diferentes dietas, sugerem que os estudos de cinética digestiva devem ser realizados com maior número de animais para que o erro associado a essas variações seja minimizado e esses parâmetros possam ser avaliados de forma mais segura.

As taxas de passagem de partículas do capim-elefante no rúmen-retículo $\left(\mathrm{k}_{1}\right)$ diminuíram de 4,04 para $3,76 \% / \mathrm{h}$ com o aumento do nível de fibra da dieta (Tabela 3), enquanto no ceco-cólon $\left(\mathrm{k}_{2}\right)$, em valores absolutos, foram maiores para a dieta com alta fibra $(9,96 \%)$ em relação à dieta com baixa fibra (8,59\%/h). Os tempos de retenção no rúmen-retículo (TRRR) foram 13,8\% menores para a dieta com 30\% de FDN que para a dieta com $60 \%$ de FDN. No entanto, os tempos

Tabela 2 - Regressão ajustadas da curva de excreção do marcador (Cr-mordante) em novilhas leiteiras de diferentes grupos genéticos submetidas a dietas com diferentes níveis de fibra

Table 2 - Fitted regression of the marker (Cr-mordanted) excretion curves in dairy heifers from different genetic groups fed diets with different levels of

\begin{tabular}{|c|c|c|c|}
\hline $\begin{array}{l}\text { Dieta } \\
\text { Diet }\end{array}$ & $\begin{array}{l}\text { Grupo } \\
\text { Group }\end{array}$ & $\begin{array}{l}\text { Regressão ajustada } \\
\text { Fitted regresssion }\end{array}$ & $\mathrm{R}^{2}$ \\
\hline \multirow{2}{*}{$\begin{array}{l}\text { FDN30 } \\
\text { NDF } 30\end{array}$} & $7 / 8 \mathrm{HZ}$ & $\hat{\mathrm{Y}}=2,5750 * \exp ^{(-0,0415 *(\mathrm{t}-9,6224))}-2,5750 * \exp ^{(-0,0894 *(\mathrm{t}-9,6224))}$ & 0,9860 \\
\hline & $\begin{array}{l}15 / 16 \mathrm{HZ} \\
\text { HPC }\end{array}$ & $\begin{array}{l}\hat{\mathrm{Y}}=5,3751^{*} \exp ^{(-0,0473 *(\mathrm{t}-11,6553))}-5,3751^{*} \exp ^{(-0,0770 *(\mathrm{t}-11,6553))} \\
\hat{\mathrm{Y}}=1,1744^{*} \exp ^{(-0,0324 *(\mathrm{t}-11,6550))}-1,1744^{*} \exp ^{(-0,0913 *(\mathrm{t}-11,6550))}\end{array}$ & $\begin{array}{l}0,9680 \\
0,9923\end{array}$ \\
\hline \multirow{2}{*}{$\begin{array}{l}\text { FDN60 } \\
\text { NDF } 60\end{array}$} & $7 / 8 \mathrm{HZ}$ & $\hat{\mathrm{Y}}=1,8626 * \exp ^{(-0,0355 *(\mathrm{t}-11,5146))}-1,8626 * \exp ^{(-0,1151 *(\mathrm{t}-11,5146))}$ & 0,9870 \\
\hline & $\begin{array}{l}15 / 16 \mathrm{HZ} \\
\mathrm{HPC}\end{array}$ & $\begin{array}{l}\hat{\mathrm{Y}}=1,4327^{*} \exp ^{(-0,0306 *(\mathrm{t}-11,6845))}-1,4327^{*} \exp ^{(-0,1097 *(\mathrm{t}-11,6845))} \\
\hat{\mathrm{Y}}=4,3323^{*} \exp ^{(-0,0466 *(\mathrm{t}-11,7625))}-4,3323^{*} \exp ^{(-0,0741 *(\mathrm{t}-11,7625))}\end{array}$ & $\begin{array}{l}0,9763 \\
0,9697\end{array}$ \\
\hline $\begin{array}{l}\text { FDN30 } \\
\text { NDF } 30\end{array}$ & & $\hat{\mathrm{Y}}=2,9749 * \exp ^{(-0,0404 *(\mathrm{t}-10,8928))}-2,9749 * \exp ^{(-0,0859 *(\mathrm{t}-10,8928))}$ & 0,9839 \\
\hline $\begin{array}{l}\text { FDN60 } \\
\text { NDF } 60\end{array}$ & & $\hat{\mathrm{Y}}=2,5425 * \exp ^{(-0,0376 *(\mathrm{t}-11,6538))}-2,5425 * \exp ^{(-0,0996 *(\mathrm{t}-11,6538))}$ & 0,9777 \\
\hline
\end{tabular}
fiber 
Tabela 3 - Tempo de retenção no rúmen-retículo (TRRR), tempo de retenção no ceco-cólon (TRCC), tempo médio de retenção nos dois compartimentos (TMR) e tempo de retenção total (TRT) em novilhas leiteiras de diferentes grupos genéticos submetidas a dietas com diferentes níveis de fibra

Table 3 - $\quad$ Rumen-reticulum retention time (RRRT), retention time in the cecum-colon (RTCC), mean retention time in the two compartments (RTC) and total retention time (TRT), in dairy heifers from different genetic groups submitted to diets with different levels of fiber

\begin{tabular}{|c|c|c|c|c|c|}
\hline Dieta & Grupo & TRRR (h) & TRCC (h) & TMR (h) & TRT (h) \\
\hline Diet & Group & $R R R T$ & RTCC & $R T C$ & $T R T$ \\
\hline & 7/8HZ & 24,46 & 11,51 & 35,97 & 45,59 \\
\hline FDN30 & 15/16HZ & 21,16 & 13,67 & 34,82 & 46,48 \\
\hline \multirow[t]{2}{*}{ NDF 30} & HPC & 26,37 & 14,08 & 40,46 & 51,96 \\
\hline & 7/8HZ & 31,81 & 9,16 & 40,97 & 52,49 \\
\hline FDN60 & 15/16HZ & 31,48 & 9,32 & 40,80 & 52,34 \\
\hline NDF 60 & HPC & 21,66 & 14,72 & 36,37 & 48,14 \\
\hline FDN30 & & 24,06 & 12,86 & 36,92 & 47,66 \\
\hline \multicolumn{6}{|l|}{ NDF 30} \\
\hline FDN60 & & 27,92 & 11,29 & 39,21 & 50,82 \\
\hline NDF 60 & & & & & \\
\hline
\end{tabular}

de retenção no ceco-cólon (TRCC) foram 13,9\% maiores para a dieta com 30\% de FDN. Os tempos médios de retenção (TMR) e os tempos de retenção total (TRT) estimados foram numericamente maiores para a dieta com alta fibra.

É provável que, enquanto os maiores níveis de forragens na dieta elevaram o tempo de retenção ruminal devido à taxa de degradação mais lenta da fibra, a presença de maiores quantidades de fibra nos intestinos tenham reduzido o tempo de retenção pós-ruminal por estimular os movimentos peristálticos. De fato, Shaver et al. (1988) e Ferreira et al. (1999) observaram que elevações na quantidade de fibra nos intestinos aumentaram o peristaltismo pós-ruminal.

Os dados obtidos confirmam as observações de Beauchemin \& Buchanam-Smith (1990), que, ao avaliarem o efeito de dietas com diferentes níveis de FDN para vacas holandesas, verificaram que a taxa de passagem no rúmen para o feno (56,5\% FDN) foi menor que para a silagem de alfafa (44,4\% FDN), e de Vieira et al.(1997), que, ao avaliarem a cinética digestiva para o capim-elefante cv. Mineiro, observaram que o aumento da idade de corte resultou em elevação do nível de fibra da forrageira diminuindo a taxa de passagem. Bürger et al. (2000a), trabalhando com bezerros holandeses na faixa de 200 kg e utilizando maior número e amplitude de níveis de FDN nas dietas (62,43; 52,29; 41,23; 31,43 e 20,66\%), não observaram efeito sobre as taxas de passagem e o tempo de retenção de feno de coast-cross (Cynodon dactylon) no rúmen-retículo e no ceco-cólon proximal. Utilizando diferentes proporções de silagem de milho e feno de coast-cross na ração de novilhos castrados com 523 kg de peso, Cavalcante et al. (2004) não observaram resposta significativa das taxas de passagem de partículas no rúmen à variação da concentração de FDN na dieta.

Houve ainda diferença de 14,75\% para os tempos de retenção no rúmen-retículo entre os animais $7 / 8 \mathrm{HZ}$ e os animais HPC, de modo que os tempos, em valores absolutos, foram maiores para o primeiro grupo. Os animais 15/16HZ apresentaram valores absolutos intermediários em todas as observações.

Não houve interação significativa $(\mathrm{P}>0,05)$ grupos genéticos $\times$ níveis de fibra na dieta para as variáveis de comportamento ingestivo estudadas (Tabela 4). Os tempos gastos em alimentação, ruminação, ócio e outras atividades diferiram significativamente $(\mathrm{P}<0,01)$ entre as dietas com baixa e alta fibra. As novilhas alimentadas com rações contendo 60\% de FDN despenderam 28,0; 15,8; e 20,2\% mais de tempo com atividades de alimentação, ruminação e mastigação total, respectivamente, que aquelas alimentadas com dietas contendo 30\% de FDN. Os valores confirmam as observações de Beauchemin (1991), Dado \& Allen (1995) e Bürger et al. (2000b), que observaram aumento linear no tempo médio de alimentação e ruminação à medida que os níveis de FDN da dieta foram elevados.

Os animais alimentados com dietas com 60\% de FDN apresentaram menores $(\mathrm{P}<0,01)$ taxas de ingestão e de ruminação quando os resultados foram expressos em $\mathrm{kgMS} / \mathrm{h}$. Entretanto, nos animais deste tratamento, a taxa de ruminação foi maior quando os resultados foram expressos em kgFDN/h (Tabela 4).

O aumento do nível de FDN na dieta de 30 para 60\% elevou $(\mathrm{P}<0,05)$ o número de mastigações por bolo, o número de bolos por dia e, conseqüentemente, o número de mastigações por dia e o tempo de mastigação total, entretanto, não influenciou o tempo de mastigação por bolo e o número de mastigações por minuto (Tabela 4).

Bürger et al. (2000b), avaliando o comportamento mastigatório de bezerros holandeses alimentados com diferentes níveis de concentrado na dieta encontraram tempo médio de ruminação por bolo, de 57,20 e 55,78 segundos, respectivamente, para níveis de FDN de 62,43 e 31,43\% na ração.

Não houve diferença entre os grupos genéticos $(\mathrm{P}>0,05)$ para tempo despendido com ruminação, ócio e outras atividades (Tabela 5), entretanto, as novilhas HPC despenderam maior tempo com alimentação $(\mathrm{P}<0,05)$ em comparação às $7 / 8 \mathrm{HZ}$ e o tempo de alimentação dos animais $15 / 16 \mathrm{HZ}$ não diferiu dos demais grupos genéticos.

Apesar de os grupos genéticos não terem apresentado diferenças $(\mathrm{P}>0,05)$ quanto à taxa de ruminação, a taxa de 
Tabela 4 - Comportamento ingestivo e caracterização da mastigação merícica em novilhas leiteiras de diferentes grupos genéticos submetidas a dietas com diferentes níveis de fibra

Table 4 - Ingestive behavior and rumination chew caracterization in dairy heifers from different genetic groups submitted to diets with different levels of fiber

\begin{tabular}{lcc}
\hline Item & \multicolumn{2}{c}{ Dieta } \\
& \multicolumn{2}{c}{ Diet } \\
\cline { 2 - 3 } & FDN30 & FDN60 \\
& NDF30 & NDF60 \\
\hline
\end{tabular}

Tempo gasto (h/dia)

Spending time (h/day)

\begin{tabular}{lcc}
\hline $\begin{array}{l}\text { Alimentação } \\
\text { Feeding }\end{array}$ & $3,53 \mathrm{~b}$ & $4,90 \mathrm{a}$ \\
$\begin{array}{l}\text { Ruminação } \\
\text { Rumination }\end{array}$ & $7,23 \mathrm{~b}$ & $8,59 \mathrm{a}$ \\
$\begin{array}{l}\text { Ócio } \\
\begin{array}{l}\text { Idle } \\
\text { Outras atividades } \\
\text { Other activities }\end{array}\end{array}$ & $8,17 \mathrm{a}$ & $6,43 \mathrm{~b}$ \\
\hline
\end{tabular}

Comportamento ingestivo

Ingestive behavior

\begin{tabular}{|c|c|c|}
\hline $\begin{array}{l}\text { Taxa de ingestão }(\mathrm{kg} \mathrm{MS} / \mathrm{h}) \\
\text { Intake rate }(\mathrm{kg} D M / \mathrm{h})\end{array}$ & $2,98 a$ & $2,21 b$ \\
\hline Taxa de ruminação (kg MS/h) & $1,45 a$ & $1,25 b$ \\
\hline $\begin{array}{l}\text { Rumination rate }(\mathrm{kg} D M / \mathrm{h}) \\
\text { Taxa de ruminação }(\mathrm{kg} \text { FDN/h) } \\
\text { Rumination rate }(\mathrm{kg} N D F / \mathrm{h})\end{array}$ & $0,377 b$ & $0,723 a$ \\
\hline \multicolumn{3}{|l|}{$\begin{array}{l}\text { Mastigação merícica } \\
\text { Rumination chewing }\end{array}$} \\
\hline $\begin{array}{l}\text { № mastigações/bolo } \\
N^{o} \text { chewing/bolus }\end{array}$ & $52,39 b$ & $54,90 \mathrm{a}$ \\
\hline $\begin{array}{l}\text { Tempo mastigação/bolo (seg.) } \\
\text { Chewing time/bolus }\end{array}$ & 48,98 & 49,60 \\
\hline $\begin{array}{l}\text { № mastigações/min } \\
\text { N. chewing/min }\end{array}$ & 64,41 & 66,74 \\
\hline $\begin{array}{l}\text { № mastigações/dia } \\
\text { N. chewing/day }\end{array}$ & $28.446,72 b$ & $35.102,51 \mathrm{a}$ \\
\hline $\begin{array}{l}\text { № de bolos/dia } \\
N \text {. bolus/day }\end{array}$ & $542,98 b$ & $636,39 a$ \\
\hline $\begin{array}{l}\text { Tempo de mastigação total (h/dia) } \\
\text { Total chewing time (h/day) }\end{array}$ & $10,76 b$ & $13,49 a$ \\
\hline
\end{tabular}

Médias na mesma linha seguidas de letras diferentes diferem $(P<0,05)$ pelo teste $\mathrm{F}$.

Means followed by different letters within a row differ $(P<0.05)$ by F test.

ingestão das novilhas HPC foi, em média, 18,2\% menor $(\mathrm{P}<0,01)$ que a encontrada para as novilhas $7 / 8 \mathrm{HZ}$ e $15 / 16 \mathrm{HZ}$ e o tempo de mastigação total foi $6,86 \%$ maior para o primeiro grupo, indicando que há diferenças etológicas entre os grupos. Polli et al. (1996) observaram que bovinos e bubalinos não apresentaram diferenças significativas quanto ao número de mastigações merícicas por bolo e o tempo de mastigação por bolo, no entanto, o número de mastigações merícicas por dia e o número de bolos por dia foi maior para o primeiro grupo.

O tempo de mastigação por bolo foi maior $(\mathrm{P}<0,05)$ para as novilhas 7/8HZ em relação às 15/16HZ, entretanto, foram
Tabela 5 - Comportamento ingestivo e caracterização da mastigação merícica em novilhas leiteiras de diferentes grupos genéticos

Table 5 - Ingestive behavior and rumination chew caracterization in dairy heifers from different genetic groups

\begin{tabular}{|c|c|c|c|}
\hline \multirow[t]{2}{*}{ Item } & \multicolumn{3}{|c|}{$\begin{array}{l}\text { Grupo genético } \\
\text { Genetic group }\end{array}$} \\
\hline & $7 / 8 \mathrm{HZ}$ & 15/16 HZ & HPC \\
\hline \multicolumn{4}{|c|}{$\begin{array}{l}\text { Tempo gasto em ( } \mathrm{h} / \text { dia) } \\
\text { Spending time (h/day) }\end{array}$} \\
\hline $\begin{array}{l}\text { Alimentação } \\
\text { Feed }\end{array}$ & $3,98 b$ & $4,04 \mathrm{ab}$ & $4,61 \mathrm{a}$ \\
\hline $\begin{array}{l}\text { Ruminação } \\
\text { Rumination }\end{array}$ & 7,71 & 8,10 & 7,93 \\
\hline Ócio (Idle) & 7,58 & 7,28 & 7,04 \\
\hline $\begin{array}{l}\text { Outras atividades } \\
\text { Other activities }\end{array}$ & 4,74 & 4,57 & 4,42 \\
\hline
\end{tabular}

Comportamento ingestivo

Ingestive behavior

\begin{tabular}{llcc}
\hline $\begin{array}{l}\text { Taxa de ingestão }(\mathrm{kg} \mathrm{MS} / \mathrm{h}) \\
\text { Intake rate }(\mathrm{kg} D M / \mathrm{h})\end{array}$ & $2,75 \mathrm{a}$ & $2,78 \mathrm{a}$ & $2,26 \mathrm{~b}$ \\
$\begin{array}{l}\text { Taxa de ruminação } \\
\text { MS }(\mathrm{kg} \mathrm{MS} / \mathrm{h})\end{array}$ & 1,36 & 1,35 & 1,30 \\
$\begin{array}{l}\text { Rumination rate }(\mathrm{kg} D M / \mathrm{h}) \\
\text { Taxa de ruminação }\end{array}$ & 0,569 & 0,547 & 0,536 \\
$\begin{array}{l}\text { FDN }(\mathrm{kg} \text { FDN/h) } \\
\text { Rumination rate }(\mathrm{kg} N \mathrm{~N} / \mathrm{h})\end{array}$ & & & \\
\hline
\end{tabular}

Rumination rate $(\mathrm{kg} N \mathrm{NF} / \mathrm{h})$

Mastigação merícica

Rumination chew

\begin{tabular}{lrrr}
\hline $\begin{array}{l}\text { № Mastigações/bolo } \\
N \text {. chewing/bolus }\end{array}$ & 53,73 & 53,34 & 53,87 \\
$\begin{array}{l}\text { Tempo mastigação/ } \\
\text { bolo (seg.) }\end{array}$ & $51,06 \mathrm{a}$ & $47,62 \mathrm{~b}$ & $49,19 \mathrm{ab}$ \\
$\begin{array}{l}\text { Chewing time/bolus } \\
\text { № de mastigações/min }\end{array}$ & $63,22 \mathrm{~b}$ & $67,49 \mathrm{a}$ & $66,03 \mathrm{ab}$ \\
$\begin{array}{l}\text { N. chewing/min. } \\
\text { № mastigações/dia }\end{array}$ & $2.9670,24 \mathrm{~b}$ & $3.3282,03 \mathrm{a}$ & $1.937,91 \mathrm{ab}$ \\
$\begin{array}{l}\text { N. chewing/day } \\
\text { № de bolos/dia }\end{array}$ & $552,21 \mathrm{~b}$ & $623,96 \mathrm{a}$ & $592,87 \mathrm{ab}$ \\
$\begin{array}{l}\text { N. bolus/day } \\
\text { Tempo de mastigação } \\
\text { total (h/dia) }\end{array}$ & 11,68 & 12,14 & 12,54 \\
Total chewing time (h/day) & & & \\
\end{tabular}

Médias na mesma linha seguidas de letras diferentes diferem $(P<0,05)$ pelo teste $\mathrm{F}$.

Means followed by different letters within a row differ $(P<0.05)$ by $F$ test.

observados menores valores $(\mathrm{P}<0,05)$ para número de mastigações por minuto, número de mastigações por dia e número de bolos por dia para os animais do grupo 7/8 HZ em relação aos do grupo 15/16HZ. Para estas variáveis os valores observados para as novilhas HPC foram intermediários entre os dos demais grupos genéticos. Não houve diferença entre os grupos $(\mathrm{P}>0,05)$ para o número de mastigações por bolo e o tempo de mastigação total.

Entre as dietas com baixo e alto nível de fibra, houve diferença $(\mathrm{P}<0,01)$ para todas as variáveis relacionadas com o comportamento ingestivo e mastigatório quando medidas em relação ao consumo de MS e de fibra na ração (Tabela 6). 
Tabela 6 - Comportamento ingestivo e mastigatório em relação ao consumo de MS e de FDN em novilhas leiteiras submetidas a dietas com diferentes níveis de fibra

Table 6 - $\quad$ Ingestive and chew behavior in relation to dry matter and fiber (NDF) intake for dairy heifers submitted to diets with different levels of fiber

\begin{tabular}{lrr}
\hline Item & \multicolumn{2}{c}{ Dieta } \\
\cline { 2 - 3 } Diet \\
\cline { 2 - 3 } & FDN30 & FDN60 \\
& NDF30 & NDF60
\end{tabular}

Comportamento ingestivo (h/kg MS)

Ingestive behavior ( $h / \mathrm{kg} D M)$

\begin{tabular}{lcc}
\hline $\begin{array}{l}\text { Taxa de ingestão } \\
\text { Intake rate }\end{array}$ & $0,3505 \mathrm{~b}$ & $0,4607 \mathrm{a}$ \\
$\begin{array}{l}\text { Taxa de ruminação } \\
\text { Rumination rate }\end{array}$ & $0,7192 \mathrm{~b}$ & $0,8090 \mathrm{a}$ \\
$\begin{array}{l}\text { Taxa de mastigação total } \\
\text { Total chewing rate }\end{array}$ & $1,0697 \mathrm{~b}$ & $1,2697 \mathrm{a}$ \\
\end{tabular}

Comportamento ingestivo (h/kg FDN)

Ingestive behavior ( $h / \mathrm{kg} N D F$ )

\begin{tabular}{lcc}
\hline Taxa de ingestão & $1,3287 \mathrm{a}$ & $0,8015 \mathrm{~b}$ \\
$\begin{array}{l}\text { Intake rate } \\
\text { Taxa de ruminação }\end{array}$ & $2,7357 \mathrm{a}$ & $1,4061 \mathrm{~b}$ \\
$\begin{array}{l}\text { Rumination rate } \\
\text { Taxa de mastigação total }\end{array}$ & $4,6440 \mathrm{a}$ & $2,2076 \mathrm{~b}$ \\
$\begin{array}{l}\text { Total chewing rate } \\
\text { Taxa ruminação por kg FDN/ }\end{array}$ & $0,11 \mathrm{a}$ & $0,05 \mathrm{~b}$ \\
$\begin{array}{l}\text { tempo médio de retenção } \\
\text { ruminal dA FDN }\end{array}$ & & \\
$\begin{array}{l}\text { Rumination rate per } k g \text { NDF/ } \\
\text { average ruminal retention } \\
\text { time of } N D F\end{array}$ & & \\
$\begin{array}{l}\text { Taxa de ruminação/tempo } \\
\text { mastigação total } \% / \mathrm{h}) \\
\text { Rumination rate/total time chewing }(\% / h)\end{array}$ & $67,3062 \mathrm{a}$ & $63,7192 \mathrm{~b}$ \\
\hline
\end{tabular}

Médias na mesma linha seguidas de letras diferentes diferem $(P<0,05)$ pelo teste $\mathrm{F}$.

Means followed by different letters within a row differ $(P<0.05)$ by $F$ test.

As taxas de ingestão, ruminação e mastigação (h/kgMS) foram maiores $(\mathrm{P}<0,01)$ para a dieta com nível mais elevado de fibra. Por outro lado, o tempo para execução das mesmas atividades, ajustadas por unidade de fibra consumida (h/kgFDN), foi menor para a dieta com $60 \%$ de FDN em relação à dieta com $30 \%$.

O tempo de ruminação por kg de FDN consumido de acordo com o tempo de retenção da ração no rúmen-retículo, assim como a porcentagem da ruminação em relação ao tempo de mastigação total, foi maior para a ração com menor nível de volumoso. Assim, pode-se observar que houve maior eficiência da quebra de partículas do volumoso para a dieta com menor nível de fibra.

Polli et al. (1996), comparando dietas à base de silagem de milho e cana-de-açúcar, observaram diferença entre os volumosos para taxa média de ruminação, de 1,04 para $1,45 \mathrm{~h} / \mathrm{kg}$ de MS ingerida, respectivamente, e maior tempo de mastigação para a cana, em razão de seu maior conteúdo de fibra. Os valores de comportamento ingestivo encontrados por esses autores para dietas com $70 \%$ de volumoso foram similares aos obtidos neste estudo para a dieta com $60 \%$ de FDN.

Os dados obtidos corroboram os de Shaver et al. (1988), que descreveram diminuição no tempo de ruminação por unidade de fibra consumida de acordo com o aumento do consumo de MS. Beauchemin \& BuchananSmith (1990) encontraram tempo médio de ruminação de 1,15 h/kg de FDN em dietas contendo 17\% de FDA, independentemente da composição da dieta e do método de alimentação.

Como a ruminação é necessária para redução no tamanho de partícula da fibra e a taxa de ruminação da FDN diminuiu $(\mathrm{P}<0,05)$ com o aumento do conteúdo de fibra da dieta, os tempos de ruminação e mastigação por unidade de FDN consumida decresceram. Resultados similares foram observados por Jung \& Allen (1995) e Albright (1993), que descreveram redução no tempo de ruminação por unidade de FDN, 1,25 vs 1,15 h/kg FDN ao suplementarem dietas com feno para vacas leiteiras.

Além disso, de acordo com Dado \& Allen (1995), a maior taxa de ingestão da MS para dietas com baixa fibra está relacionada ao aumento na taxa de passagem da fibra nos compartimentos ruminais e, conseqüentemente, ao menor tempo de retenção ruminal da FDN.

Apesar de não terem sido observadas diferenças entre os grupos genéticos para as taxas de ruminação, as novilhas HPC apresentaram maiores taxas de ingestão $(P<0,01)$ e mastigação $(\mathrm{P}<0,05)$, por unidade de MS e por unidade de FDN consumida, em comparação às novilhas $7 / 8 \mathrm{HZ}$. O grupo HPC apresentou ainda menor taxa de ruminação em relação ao tempo de mastigação total, em razão do maior tempo gasto em alimentação e da menor taxa de ingestão (Tabela 7).

\section{Conclusões}

Quando o capim-elefante é utilizado como único volumoso, níveis de $60 \%$ de FDN na ração, em comparação a $30 \%$, resultam em maior tempo de retenção, menor taxa de passagem de partículas no rúmem-retículo e menor tempo de retenção no ceco-cólon.

Novilhas alimentadas com dietas contendo $60 \%$ de FDN despendem mais tempo com alimentação e ruminação, apresentam maior taxa de ruminação por kg de FDN, maior número de mastigações por bolo, maior número de mastigações merícicas por dia, maior número de bolos e maior tempo de mastigação por dia em comparação àquelas alimentadas com dietas contendo 30\% de FDN. 
Tabela 7 - Comportamento ingestivo e mastigatório em relação ao consumo de matéria seca e de FDN para novilhas leiteiras de diferentes grupos genéticos

Table 7 - Ingestive and chewing behavior in relation to dry matter and NDF intake for dairy heifers from different genetic groups

\begin{tabular}{lccc}
\hline Item & \multicolumn{3}{c}{$\begin{array}{c}\text { Grupo genético } \\
\text { Genetic group }\end{array}$} \\
\cline { 2 - 4 } & $7 / 8 \mathrm{HZ}$ & $15 / 16 \mathrm{HZ}$ & $\mathrm{HPC}$ \\
\hline $\begin{array}{l}\text { Comportamento ingestivo } \\
\text { (h/kg MS) }\end{array}$ & & & \\
$\begin{array}{l}\text { Intake behavior ( } \mathrm{h} / \mathrm{kg} \mathrm{DM}) \\
\text { Taxa de ingestão }\end{array}$ & $0,3831 \mathrm{~b}$ & $0,3777 \mathrm{~b}$ & $0,4560 \mathrm{a}$ \\
$\begin{array}{l}\text { Intake rate } \\
\text { Taxa de ruminação } \\
\text { Rumination rate }\end{array}$ & 0,7478 & 0,7602 & 0,7842 \\
$\begin{array}{l}\text { Taxa de mastigação total } \\
\text { Total chewing rate }\end{array}$ & $1,1310 \mathrm{~b}$ & $1,1379 \mathrm{~b}$ & $1,2402 \mathrm{a}$ \\
\hline
\end{tabular}

Comportamento ingestivo (h/kg FDN)

Intake behavior $(\mathrm{h} / \mathrm{kg} N D F)$

\begin{tabular}{|c|c|c|c|}
\hline Taxa de ingestão & $0,9928 b$ & $0,9764 b$ & $1,2261 \mathrm{a}$ \\
\hline Intake rate & & & \\
\hline $\begin{array}{l}\text { Taxa de ruminação } \\
\text { Rumination rate }\end{array}$ & 2,0341 & 2,0350 & 2,1437 \\
\hline $\begin{array}{l}\text { Taxa de mastigação total } \\
\text { Total chewing rate }\end{array}$ & $3,0268 b$ & $3,0114 b$ & $3,3698 a$ \\
\hline $\begin{array}{l}\text { Taxa ruminação por kg FDN/ } \\
\text { tempo médio de retenção } \\
\text { ruminal do FDN }\end{array}$ & 0,0885 & 0,0855 & 0,0790 \\
\hline $\begin{array}{l}\text { Rumination rate per } \mathrm{kg} N D F / \\
\text { average ruminal retention } \\
\text { time of } N D F\end{array}$ & & & \\
\hline $\begin{array}{l}\text { Taxa de ruminação/ } \\
\text { tempo mastigação total }(\% / \mathrm{h}) \\
\text { Rumination rate/total time chew }(\%\end{array}$ & $\begin{array}{l}66,3167 a \\
\text { /h) }\end{array}$ & $66,9995 a$ & $63,2218 b$ \\
\hline
\end{tabular}

Médias na mesma linha seguidas de letras diferentes diferem $(P<0,05)$ pelo teste $\mathrm{F}$.

Means followed by different letters within a row differ $(P<0.05)$ by F test.

Novilhas do grupo genético Holandês PC despendem maior tempo com atividade de alimentação quando comparadas a novilhas $7 / 8 \mathrm{HZ}$, entretanto, o grupo genético não influencia os tempos utilizados para ruminação, ócio e outras atividades.

\section{Literatura Citada}

ALBRIGHT, J.L. Feeding behavior of dairy cattle. Journal of Dairy Science, v.76, n.2, p.485-498, 1993.

ALLEN, M. Fiber requirements: finding an optimum can be confusing. Feedstuffs, v.67, n.19, p.13, 1995.

ALLEN, M.S. Effects of diet on short-term regulation of feed intake by lactating dairy cows. Journal of Dairy Science, v.83, p.1598-1624, 2000.

BEAUCHEMIN, K.A.; BUCHANAN-SMITH, J.G. Effects of fiber source and method of feeding on chewing activities, digestive function, and productivity of dairy cows. Journal of Dairy Science, v.73, p.749-762, 1990.

BEAUCHEMIN, K.A. Effects of dietary neutral detergent fiber concentration and alfafa hay quality on chewing activities, rumen function, and milk production of dairy cows. Journal of Dairy Science, v.74, p.3140-3151, 1991.
BEZERRA, E.S.; QUEIROZ, A.C.; BEZERRA, A.R.G.F. et al. Perfil granulométrico da fibra dietética sobre o tempo médio de retenção e a digestibilidade aparente de dietas de vacas leiteiras. Revista Brasileira de Zootecnia, v.33, n.6, p.2378-2386, 2004 (supl. 3).

BÜRGUER, P.J.; PEREIRA, J.C.; COELHO DA SILVA, J.F. et al. Taxas de passagem e cinética de degradação ruminal em bezerros holandeses alimentados com dietas contendo diferentes níveis de concentrado. Revista Brasileira de Zootecnia, v.29, n.1, p.225-235, 2000a.

BÜRGUER, P.J.; PEREIRA, J.C.; QUEIROZ, A.C. et al. Comportamento ingestivo em bezerros holandeses alimentados com dietas contendo diferentes níveis de concentrado. Revista Brasileira de Zootecnia, v.29, n.1, p.236-242, 2000b.

CAVALCANTE, A.C.R.; PEREIRA, O.G.; VALADARES FILHO, et al. Dietas contendo silagem de milho (Zea maiz L.) e feno de capim-tifton 85 (Cynodon sp.) em dietas para bovinos. Revista Brasileira de Zotecnia, v.33, n.6, p.2394-2402, 2004 (supl. 3).

COLUCCI, P.E.; MACLEOD, G.K.; GROVUM, W.L. et al Digesta kinetics in sheep and cattle fed diets with different forage to concentrate ratios at high and low intakes. Journal of Dairy Science, v.73, n.8, p.2143-2156, 1990.

CRUICKSHANK, G.J.; POPPI, P.D.; SYKES, A.R. Theoretical considerations in the estimation of fractional outflow rate from various sampling sites. British Journal of Nutrition, v.62, p.229-239, 1989.

DADO, R.G.; ALLEN, M.S. Intake limitation, feeding behavior and rumen function of cows challenged with rumen fill from dietary fiber or inert bulk. Journal of Dairy Science, v.78, n.1, p.118133, 1995

EUCLYDES, R.F. Sistema para análises estatísticas e genéticas. Manual de utilização do SAEG. Viçosa, MG: Universidade Federal de Viçosa, 1983. 57p.

FERREIRA, L.A.M.; FONTES, C.M.G.A.; FERNANDES, T.H. A fibra em nutrição animal. Revista Portuguesa de Ciências Veterinárias, v.94, n.531, p.119-130, 1999.

GROVUM, W.L.; WILLIAMS, V.J. Rate of passage of digesta in sheep. 4. Passage of marker through the alimentary tract and biological relevance of rate- constants derived from the changes in concentration of marker in feces. British Journal of Nutrition, v.30, n.2, p.313-329, 1973.

JOHNSON, T.R.; COMBS, D.K. Effects of prepartum diet, inert rumen bulk, and dietary polyethylene glycol on dry matter intake of lactating dairy cows. Journal of Dairy Science, v.74, n.3, p.933-944, 1991.

JUNG, H.G.; ALLEN, M.S. Characteristics of plant cell walls affecting intake and digestibility of forages by ruminants. Journal of Animal Science, v.73, .2774-2790, 1995.

MERTENS, D.R. Creating a system for meeting the fiber requirements of dairy cows. Journal of Dairy Scence, v.80, p.1463-1481, 1997.

NATIONAL RESEARCH COUNCIL - NRC. Nutrient requirements of dairy cattle. Washington, D.C.: National Academy of Sciences, 2001. 363p.

NATIONAL RESEARCH COUNCIL - NRC. Nutrient requirements of dairy cattle. Washington, D.C.: National Academy of Sciences, 1989. 157p.

POLLI, V.A.; RESTLE, J.; SENNA, D.B. et al. Aspectos relativos à ruminação de bovinos e bubalinos em regime de confinamento. Revista Sociedade Brasileira de Zootecnia, v.25, n.5, p.987-993, 1996.

SARMENTO, D.O.L. Comportamento ingestivo de bovinos em pastos de capim-marandu submetidos a regimes de lotação contínua. Piracicaba: Escola Superior de Agricultura Luiz de Queiroz, 2003. 76p. Dissertação (Mestrado em Ciência Animal e Pastagem) - Escola Superior de Agricultura Luiz de Queiroz, 2003.

SEJRSEN, K.; FOLDAGER, J. Mammary growth and milk production capacity of replacement heifers in relation to diet energy 
concentration and plasma hormone levels. Acta Agricultural Scandinavica- Section Animal Science, v.42, p.99, 1992.

SHAVER, R.D.; NYRTES, J. ; SATTER, L.D. et al. Influence od feed intake, forage physical form and forage fiber content on particle size of masticated forage, ruminal digesta and feces of dairy cows. Journal of Dairy Science, v.71, p.1566-1572, 1988.

SHAFFER, L.; ROUSSEL, J.D.; KOONCE, K.L. Effects of age, temperature- season, and breed on blood characteristics of dairy cattle. Journal of Dairy Science, v.64, n.1, p.62-70, 1981.

SILVA, D.J. Análise de alimentos (métodos químicos e biológicos). 2.ed. Viçosa, MG: Editora UFV, 2002. 165p.

TOMLINSON, D.J.; JAMES, R.E.; MCGILLIARD, E.D. Effect of varying levels of neutral detergent fiber and total digestible nutrients on intake and growth of Holstein heifers. Journal of Dairy Science, v.74, p.537-545, 1991.

ÚDEN, P.; COLUCCI, P.E.; van SOEST, P.J. Investigation of chromium, cerium, and cobalt as markers in digesta rate of passage studies. Journal of Science Food and Agricultural, v.31, n.6, p.625-632, 1980.
Van SOEST, P.J. Nutritional ecology of the ruminant. 2.ed. Ithaca: Cornell University, 1994. 476p.

Van SOEST, P.J.; ROBERTSON, J.B.; LEWIS, B.A. Methods for dietary fiber, neutral detergent fiber, and nonstarch polysaccharides in relation to animal nutrition. Journal of Dairy Science, v.74, p.3583-3597, 1991.

VIEIRA, R.A.M.; PEREIRA, J.C.; MALAFAIA, P.A.M. et al. The influence of elephantgrass Pennisetum purpureum, Schum., Mineiro variety) growth on the nutrients kinetics in the rumen. Animal Feed Science and Technology, v.67, p. 151-161, 1997. WILLIAMS, C.H.; DAVID, D.J.; IISMAA, O. The determination of chromic oxide in faeces samples by atomic absorption spectrophotometry. Journal of Agricultural Science, v.59, p.381-385, 1962. 